\title{
The role of lumbar puncture and pressure-lowering therapy for transverse dural sinus thrombosis
}

\author{
Joshua Grant, Andrew Carey, Curtis Margo, Reed Murtagh, Mitchell Drucker \\ Departments of Ophthalmology and Radiology, Morsani College of Medicine, University of South Florida, Tampa, FL, USA
}

\section{ABSTRACT}

Transverse (lateral) sinus thrombosis is a well-known complication of acute otitis media and mastoiditis in the pediatric and adult population. Thrombosis involving the transverse sinus can ultimately cause elevation of intracranial pressure (ICP) as a result of decreased cerebrospinal fluid absorption. If treatment to lower ICP is not undertaken, it can lead to ophthalmological complications including irreversible vision loss. The following case report describes an 11-year-old girl who was diagnosed with AOM by her pediatrician and subsequently presented to the emergency department complaining of nausea, vomiting, headache, and diplopia.

Key words: Acute otitis media, cranial nerve VI palsy, diplopia, increased intracranial pressure, sinus thrombosis

\section{Introduction}

Transverse dural sinus thrombosis is a well-known complication of acute otitis media (AOM) and mastoiditis, accounting for $17-19 \%$ of all intracranial complications. ${ }^{[1]}$ Secondary elevation of intracranial pressure (ICP) can cause neurological and visual symptoms, prompting the patient to seek medical evaluation. Computed tomography (CT), magnetic resonance imaging (MRI), and magnetic resonance venography (MRV) are essential in establishing a timely diagnosis. Lumbar puncture (LP) with opening pressure and cerebral spinal fluid (CSF) analysis are just as crucial in directing the optimal management strategy. The following case report describes an 11-year-old girl with AOM who presented to the emergency department (ED) complaining of nausea, vomiting, headache, and diplopia, and highlights the role of baseline LP and prompt pressure-lowering therapy to minimize morbidity.

\begin{tabular}{|l|l|}
\hline \multicolumn{2}{|c|}{ Access this article online } \\
\hline Quick Response Code: & Website: \\
\hline & www.ruralneuropractice.com \\
\cline { 2 - 3 } & \\
\hline & \\
\hline
\end{tabular}

\section{Case Report}

An 11-year-old girl with no significant past medical history presented to the ED complaining of 2 days of worsening headache, vomiting, and double vision. Approximately 2 weeks earlier, the patient was seen by her primary care physician (PCP) for evaluation of flu-like symptoms, headache, and left-sided neck pain. Examination at that time revealed an erythematous right tympanic membrane. The patient was diagnosed with AOM and started on oral amoxicillin/clavulanate. The patient continued to complain of worsening headache for 7 days following PCP evaluation, at which point she developed diplopia, nausea, and vomiting. She was subsequently taken to the ED for evaluation.

Non-contrast $\mathrm{CT}$ revealed an asymmetrically hyperdense right transverse sinus [Figure 1]. Brain MRI demonstrated a thrombus in the right transverse sinus as well as fluid within the right mastoid air cells consistent with mastoiditis [Figures 2-4]. A filling void in the right transverse sinus, consistent with a right transverse sinus thrombosis, was found on MRV [Figure 5].

Neurology evaluation did not reveal any focal abnormalities but ophthalmic examination was incomplete; LP was not recommended. The patient was admitted to the pediatric intensive care unit

Address for correspondence:

Dr. Andrew Carey, Department of Ophthalmology, USF Morsani College of Medicine, 12901 Bruce B. Downs BIvd., MDC 21, Tampa FL, USA. E-mail: acarey@health.usf.edu 


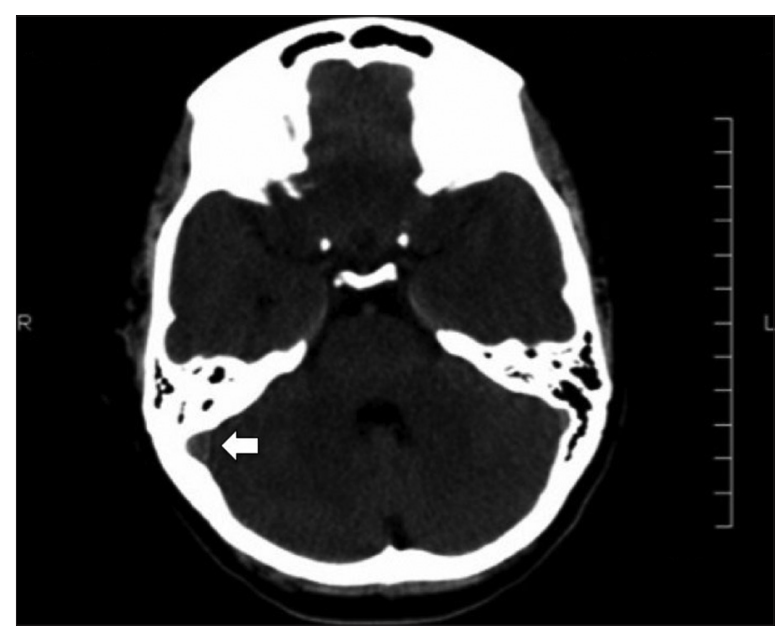

Figure 1: Non-contrast head CT without contrast demonstrating a hyperdense right transverse sinus (arrow)

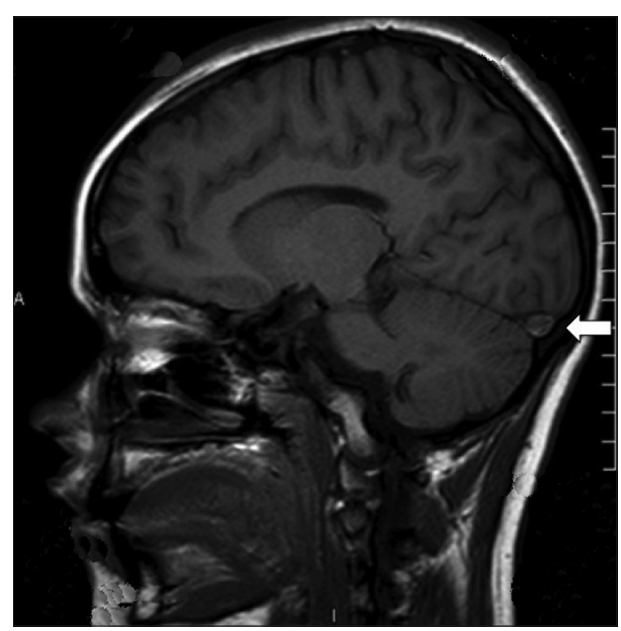

Figure 3: "Delta" sign in right transverse sinus on coronal postcontrast T1-weighted image (arrow)

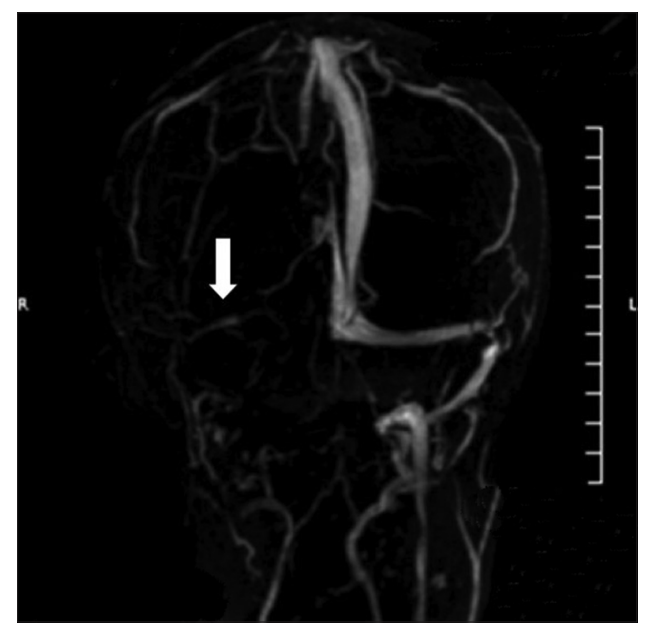

Figure 5: MRV showing absent occluded right transverse sinus (arrow)

where heparin drip and intravenous antibiotics were started. Hypercoagulablity studies were negative. Ophthalmology consultation found a small right

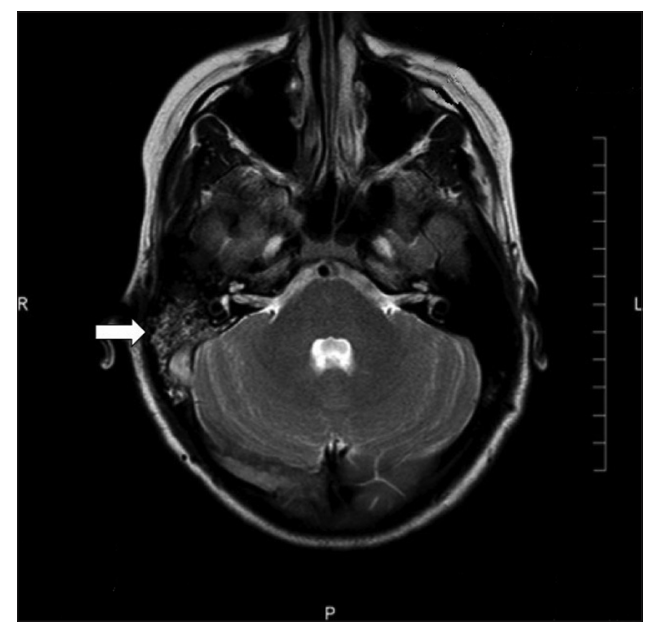

Figure 2: Axial T2-weighted image showing lack of flow in right transverse sinus adjacent to abnormal right mastoid air spaces (arrow)

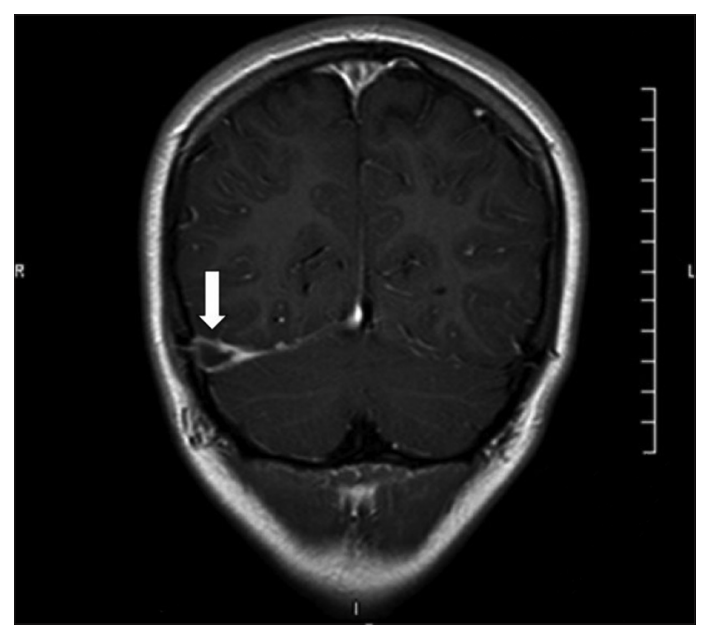

Figure 4: T1-weighted MRI showing a thrombus in the right transverse sinus (arrow)

esotropia in primary gaze; optic disc edema bilaterally; and nerve fiber layer hemorrhages more pronounced in the right fundus than left. The patient was diagnosed with papilledema and palsy of the right cranial nerve VI, secondary to increased ICP.

The patient was discharged on oral cefdinir without performing an LP or ICP-lowering therapy. Outpatient ophthalmology follow-up 2 weeks later found worsening headache, persistent papilledema, and esotropia in primary and right-gaze. Formal visual field testing revealed enlarged blind spots bilaterally. The patient was referred to the ED for LP which revealed an opening pressure of $310 \mathrm{~mm} \mathrm{H}_{2} \mathrm{O}$ (upper limit of normal $210 \mathrm{~mm}$ ); the patient was started on oral acetazolamide. CSF studies were otherwise unremarkable. Follow-up examination 2 weeks later revealed improvement in headache and complete resolution of diplopia and esotropia. 


\section{Discussion}

Acute otitis media and mastoiditis, even with appropriate antibiotic therapy, can precipitate transverse dural sinus thrombosis. The incidence of transverse sinus thrombosis as a complication of AOM, however, has decreased significantly during the antibiotic era. ${ }^{[1,2]}$

Given the close proximity of the mastoid to the transverse sinus, inflammation can propagate from the small venules of the mastoid into the vascular wall, with eventual formation of a mural thrombus. ${ }^{[1,2]}$ Imaging studies including CT, MRI and MRV are pivotal in making a diagnosis of dural venous sinus thrombosis. When used in combination, MRI and MRV are considered the most sensitive imaging modalities. ${ }^{[3]}$ Head CT, while often normal in cerebral vein and dural sinus thromboses, is useful to rule out other intracranial disorders or abnormalities that can present with similar clinical manifestations. ${ }^{[4-6]}$ Given the low sensitivity of CT imaging in patients with dural sinus thromboses as well as variable and vague symptoms, a high index of suspicion must be maintained in patients with a new headache and visual complaints.

Elevated ICP resulting from sinus thrombosis can lead to severe neurological and ophthalmic complications, which may be irreversible without prompt pressure-lowering treatment. Symptoms vary widely, ranging from isolated headache to more ominous findings such as seizures, mental status changes, focal neurological deficits, and coma. ${ }^{[7,8]}$ Ophthalmic signs are common at presentation including cranial nerve VI palsy and papilledema in 34\% and $18 \%$ of patients, respectively, as well as visual field defects. ${ }^{[8]}$ In such situations, additional imaging studies and LP are needed to establish the diagnosis, even in the presence of normal head CT.

After a diagnosis has been made, LP (in the left lateral decubitus position) is essential for obtaining an opening pressure and guiding further therapy. It is crucial to rule out meningitis and other infectious etiologies as potential underlying causes of sinus thrombosis. Any delay in treatment of elevated ICP could result in worsening ophthalmic or neurological manifestations, and possible irreversible damage to the visual pathway or brain. Patients are at risk for ischemic injury to the optic nerve, as well as ischemic or hemorrhagic cerebral infarcts, subarachnoid and subdural hemorrhages, and intraventricular hemorrhages. ${ }^{[9]}$

Treatment of the underlying cause of the dural sinus thrombosis and initiation of anticoagulation are complimentary management strategies, but neither will lower ICP acutely or decrease morbidity associated with elevated ICP. Current guidelines for treatment of dural sinus thrombosis with associated elevation of ICP suggest, but do not mandate LP, acetazolamide therapy, and CSF-shunting procedures if there is concern over threatened vision. ${ }^{[10]}$ This case demonstrates the value of LP in documenting elevated ICP and how it guides the use of pressure-lowering therapy.

\section{Conclusions}

Thrombosis of the transverse dural sinus can arise as a complication of AOM and mastoiditis in children and adults. Anatomic localization of this process is often challenging, and ophthalmic complaints are common when the thrombus is radiologically evident. ${ }^{[8]}$ Physicians must maintain a high index of suspicion, given the frequency of normal head CT imaging in this setting. Following a radiographic diagnosis, lumbar puncture with opening pressure is valuable in guiding appropriate medical management. If, however, LP is not or cannot be performed and there is suspicion of increased ICP, ICP-lowering therapy should be initiated empirically to prevent potentially irreversible damage to the visual pathway.

\section{References}

1. Smith JA, Danner CJ. Complications of chronic otitis media and cholesteatoma. Otolaryngol Clin North Am 2006;39:1237-55.

2. Bianchini C, Aimoni C, Ceruti S, Grasso DL, Martini A. Lateral sinus thrombosis as a complication of acute mastoiditis. Acta Otorhinolaryngol Ital 2008;28:30-3.

3. Lafitte F, Boukobza M, Guichard JP, Hoeffel C, Reizine D, Ille O, et al. MRI and MRA for diagnosis and follow-up of cerebral venous thrombosis (CVT). Clin Radiol 1997;52:672-9.

4. Moreno-Ramos MD, Rodríguez-Romero R, Piñero-González de la Peña P, Rodríguez-Uranga J, Monreal-Monsalve C. Noninvasive diagnosis of cerebral venous thrombosis. Radiologia 2006;48:79-86.

5. Wasay M, Azeemuddin M. Neuroimaging of cerebral venous thrombosis. J Neuroimaging 2005;15:118-28.

6. Biousse V, Ameri A, Bousser MG. Isolated intracranial hypertension as the only sign of cerebral venous thrombosis. Neurology 1999;53:1537-42.

7. Ferro JM, Canhão P, Stam J, Bousser MG, Barinagarrementería F. ISCVT Investigators. Prognosis of cerebral vein and dural sinus thrombosis: Results of the international study on cerebral vein and dural sinus thrombosis (ISCVT). Stroke 2004;35:664-70.

8. Wasay M, Dai AI, Ansari M, Shaikh Z, Roach ES. Cerebral venous sinus thrombosis in children: A multicenter cohort from the United States. J Child Neurol 2008;23:26-31.

9. Dlamini N, Billinghurst L, Kirkham FJ. Cerebral venous sinus (sinovenous) thrombosis in children. Neurosurg Clin N Am 2010;21:511-27.

10. Einhäupl K, Stam J, Bousser MG, De Bruijn SF, Ferro JM, Martinelli I, et al. EFNS guideline on the treatment of cerebral venous and sinus thrombosis in adult patients. Eur J Neurol 2010;17:1229-35.

How to cite this article: Grant J, Carey A, Margo C, Murtagh R, Drucker $\mathrm{M}$. The role of lumbar puncture and pressure-lowering therapy for transverse dural sinus thrombosis. J Neurosci Rural Pract 2013, 4(Suppl 1):s106-8.

Source of Support: Nil. Conflict of Interest: None declared. 